\title{
The safety and efficacy of phaco-sleeve irrigation- assisted hydrodissection during femtosecond laser-assisted cataract surgery
}

This article was published in the following Dove Press journal:

Clinical Ophthalmology

\author{
Yoichiro Masuda' \\ Hisaharu Iwaki \\ Akira Watanabe ${ }^{3}$ \\ Akiko Takada ${ }^{3}$ \\ Toshinori Okamoto ${ }^{4}$ \\ Kotaro Oki ${ }^{5}$ \\ Tadashi Nakano ${ }^{3}$ \\ Hiroshi Tsuneoka ${ }^{3}$ \\ 'Department of Ophthalmology, The \\ Jikei University, Katsushika Medical \\ Center, Tokyo, Japan; ${ }^{2}$ Iwaki Eye \\ Clinic, Tokyo, Japan; ${ }^{3}$ Department of \\ Ophthalmology, The Jikei University, \\ School of Medicine, Tokyo, Japan; \\ ${ }^{4}$ Nakamurabashi Eye Clinic, Tokyo, \\ Japan; ${ }^{5}$ Oki Eye Surgery Center, \\ Tokyo, Japan
}

\begin{abstract}
Femtosecond laser-assisted cataract surgery (FLACS) changes the intraoperative environment due to the generation of intracapsular gas that induces a high intracapsular volume. Manual hydrodissection $(\mathrm{mH})$ may induce high intracapsular pressure (ICP) and additional intracapsular volume, thereby leading to capsular block syndrome (CBS). Since the phaco-sleeve irrigation-assisted hydrodissection $(\mathrm{iH})$ technique is used to initially groove and split the lens and remove the intracapsular gas, this can reduce the intracapsular volume while bypassing the intracapsular lens prior to the hydrodissection. As iH uses the phaco tip to intentionally vacuum the intraocular fluid for use in inducing the irrigation jet from the sleeve side holes, the ICP cannot surpass the set irrigation pressure, thereby avoiding CBS. Using this technique, we performed FLACS without CBS in 310 cataract eyes. Our findings suggest that the $\mathrm{iH}$ technique may be beneficial for patients by preventing CBS during FLACS.
\end{abstract}

Keywords: FLACS, irrigation dynamic pressure, hydrodissection-related complication, capsular block syndrome, CBS, low invasive cataract surgery

\section{Introduction}

The risk of intraoperative capsular block syndrome (CBS) during manual hydrodissection $(\mathrm{mH})$ has been reported after using laser irradiation for lens fragmentation during femtosecond laser-assisted cataract surgery (FLACS). ${ }^{1,2}$ During laser fragmentation of the lens, intracapsular gas is produced and can potentially increase the intracapsular volume (Figure 1A). The mechanism responsible for intraoperative CBS is thought to be associated with 1) an increase in the posterior intracapsular volume caused by additional irrigation due to pressurization during $\mathrm{mH}$ that can occur if the fluid egress from the capsular space is impeded. As a result, the lens is elevated toward the anterior, "lens-capsulotomy block" occurs in accordance with the increasing resistance around the edge of the laser-cut capsulotomy, followed by the occurrence of an "irrigation trap" within the posterior intracapsular space (Figure 1B), and 2) pressurization during the $\mathrm{mH}$ inflates the posterior capsule, thereby increasing the posterior intracapsular volume (Figure 1C). As a result, posterior capsule rupture develops in accordance with the limit of the posterior capsule capacity to resist pressure (Figure 1D)..$^{3-7}$

The possible risk of CBS might be decreased during FLACS, since the femtosecond laser technology has been much improved with time. ${ }^{8}$ However, the surgeon should avoid this severe complication by an appropriate surgical technique. 
A

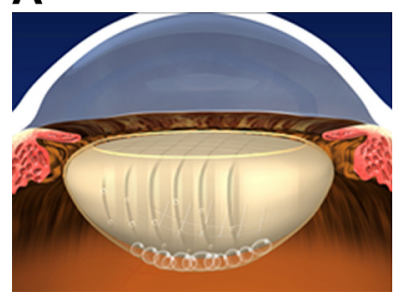

B

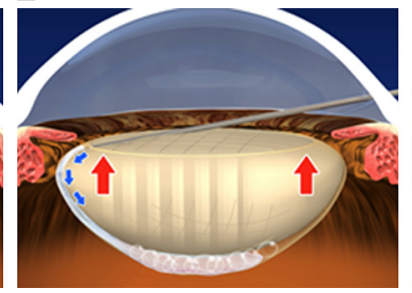

C

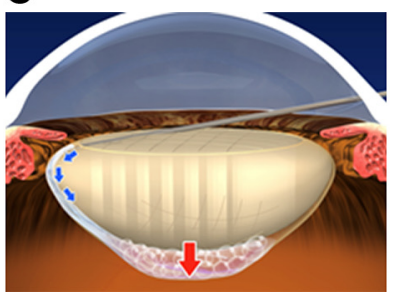

D

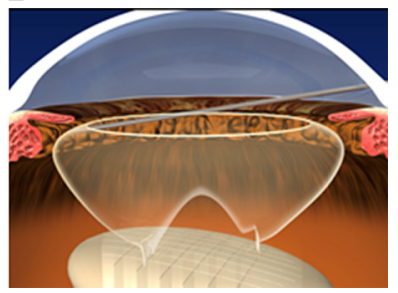

Figure I Intraoperative CBS during $\mathrm{mH}$ after femtosecond laser lens fragmentation.

Notes: (A) During femtosecond laser lens fragmentation, there is induction of intracapsular gas with a potential increase in the intracapsular volume. (B) An increase in the posterior irrigation volume due to additional intracapsular pressurization during $\mathrm{mH}$ can occur if the fluid egress from the capsular space is impeded. If this occurs, the lens will be elevated toward the anterior, followed by "lens-capsulotomy block" and "irrigation trap" within the intracapsular space. (C) Continuing pressurization by mH results in the inflation of the posterior capsule and subsequent increase in the ICP (red arrow). (D) The posterior capsule rupture can then develop during mH in accordance with the limits of the posterior capsule capacity to resist pressure. The schematic illustration shows the irrigation flow (blue arrows) and the direction of the movement of the lens segments and posterior capsule caused by the pressurized irrigation (red arrows).

Abbreviations: CBS, capsular block syndrome; ICP, intracapsular pressure; $\mathrm{mH}$, manual hydrodissection.

The phaco-sleeve irrigation-assisted hydrodissection (iH) technique (a technique that we previously reported as "irrigation dynamic pressure-assisted hydrodissection") can be used to avoid excessively high intracapsular pressures (ICPs) during cortical-capsular hydrodissection..$^{9-11}$ The $\mathrm{iH}$ technique during FLACS is initially used to groove and split the lens, followed by dislocation and aspiration of the intracapsular gas (degassing). This reduces the intracapsular volume, the front and back of the intracapsular lens can be bypassed prior to performing the hydrodissection, thereby helping to avoid "lens-capsulotomy block" and "irrigation trap" within the intracapsular space (Figure 2). Moreover, since the iH technique uses the phaco tip to intentionally vacuum the intraocular fluid in order to induce the irrigation jet from the phaco tip sleeve side hole for the use in cortical-capsular cleavage (Figure 3), this results in a reduction in the IOP and ICP from the set irrigation pressure present during the hydrodissection in the machine with bottle height-dependent irrigation pressure (Figure 4). Thus, the use of the iH technique would be expected to be beneficial
A
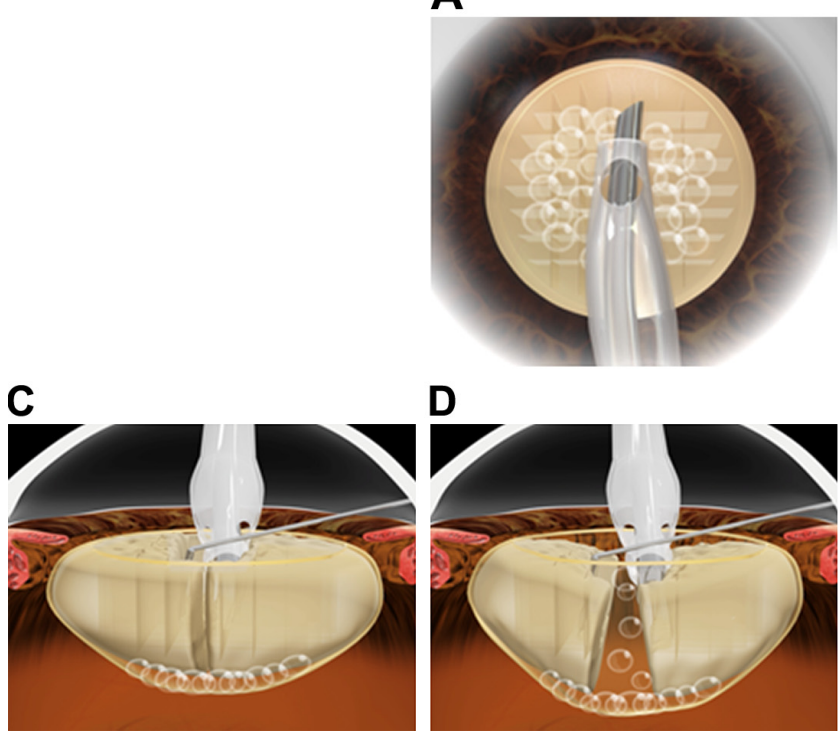

B
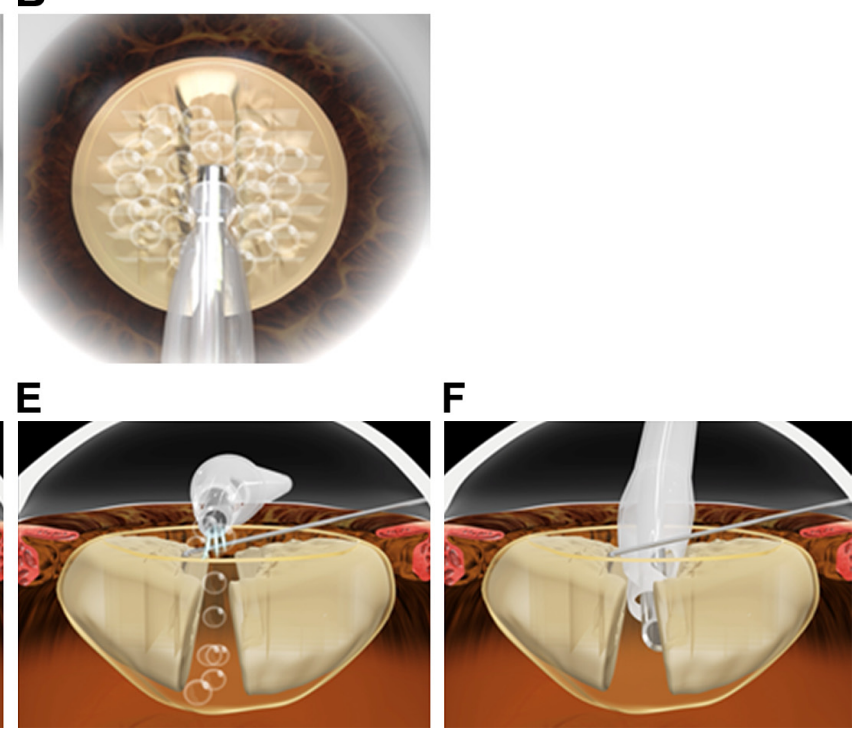

Figure 2 Bypassing the front and back of the intracapsular lens and degassing by the phaco tip and hook prior to hydrodissection induces a reduction in the intracapsular volume and pressure.

Notes: (A) The phaco tip is inserted without $\mathrm{mH}$. (B) Conventional vertical groove sculpture. (C and D) After exertion of centrifugal force from the vertical groove sculpture, the vertical groove sculpture is cracked, and the intracapsular gas is dislocated toward the anterior chamber. (E and $\mathbf{F}$ ) Since the majority of the intracapsular gas can be aspirated, this makes it possible to reduce the intracapsular volume and ICP prior to the hydrodissection. The schematic illustration shows the vacuuming of the aqueous (white lines).

Abbreviations: ICP, intracapsular pressure; $\mathrm{mH}$, manual hydrodissection. 


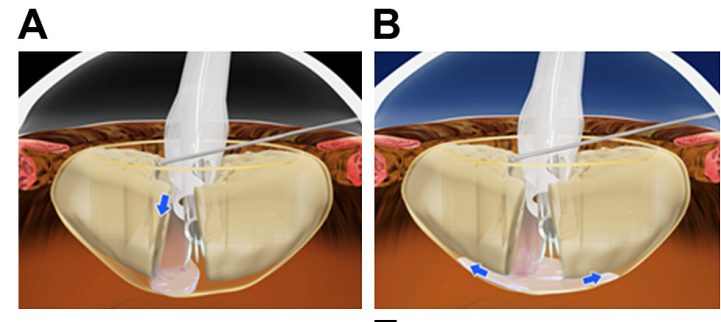

E

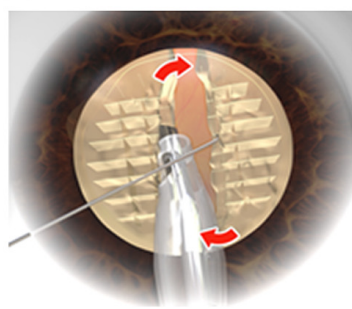

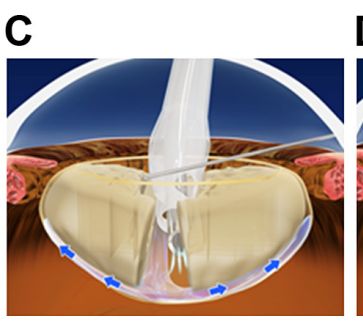

$\mathbf{F}$

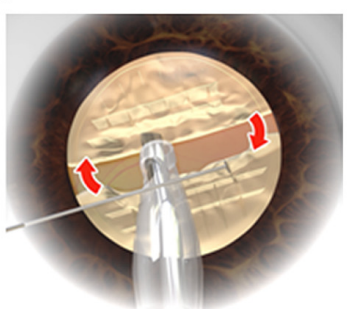

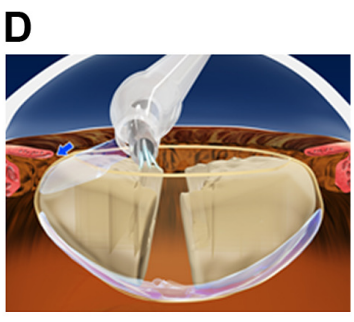

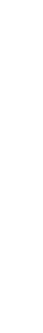

Figure 3 Performance of the iH technique after bypassing the front and back of the lens, thereby leading to a reduction in the ICP.

Notes: (A-C) Centrifugal force from the vertical groove sculpture is exerted by directing one of the sleeve side holes toward the posterior capsule, with vacuuming of the intraocular fluid using the phaco tip. This results in directing the irrigation flow jet from the phaco tip sleeve hole into the posterior cortical-capsular layers. As a result, this causes separation of the posterior cortical-capsular connection (posterior $\mathrm{iH}$ ). If the anterior cortical-capsular connection can also be separated through the use of posterior $\mathrm{iH}$, it is not necessary to perform the anterior $\mathrm{iH}$ technique. (D) If needed, anterior $\mathrm{iH}$ is performed after posterior cleavage. During this procedure, the irrigation jet from one of the sleeve side holes is directed toward the anterior cortical layer between the capsulorhexis edge and the lens with vacuuming of the intraocular fluid using the phaco tip. The irrigation jet can then be used to perform the anterior $\mathrm{iH}$. (E and $\mathbf{F}$ ) The lens can be freed from the cortical-capsular connection by using the phacoemulsification tip and hook to circumferentially rotate it for removal. The schematic illustration shows the direction of the movement of the lens segments caused by the instruments (red arrows), along with the vacuuming of the aqueous (white lines), and the irrigation flow (blue arrows).

Abbreviations: ICP, intracapsular pressure; iH, irrigation-assisted hydrodissection.

for patients, as it prevents high ICP-induced complications such as intraoperative CBS during FLACS. In this study, we further describe the use of this technique and the clinical results for the use of $\mathrm{iH}$ during FLACS.

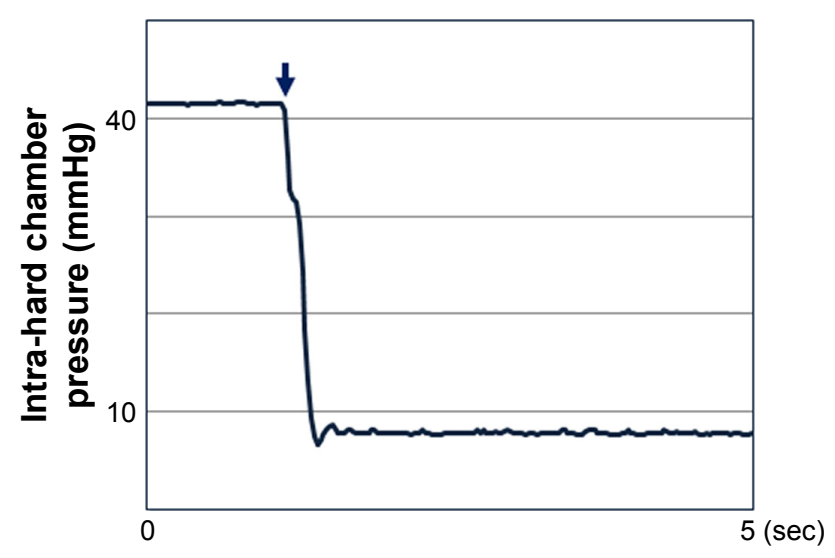

Figure 4 Depressurization during iH (experimental intra-hard chamber data in the machine with bottle height-dependent irrigation pressure).

Notes: The phaco-tip sleeve and a pressure transducer were connected to a hard chamber. Subsequently, we then examined the intra-hard chamber pressure during the $\mathrm{iH}$ procedure. The vertical axis shows the intra-hard chamber pressure $(\mathrm{mmHg})$, while the horizontal axis shows the time $(\mathrm{s})$. The arrow indicates the initial point of the $\mathrm{iH}$, with vacuuming of the intra-hard chamber fluid used to induce the irrigation jet. The graph shows that the maximum intra-hard chamber pressure was the same as the set irrigation pressure (bottle height-dependent hydrostatic pressure), whereas the intra-hard chamber pressure was reduced during the $\mathrm{iH}$ procedure.

Abbreviations: $\mathrm{iH}$, irrigation-assisted hydrodissection; s, seconds.

\section{Surgical techniques Bypassing the front and back of the intracapsular lens and reducing the intracapsular volume and ICP prior to hydrodissection}

In order to eliminate intraoperative environments that could possibly induce intraoperative $\mathrm{CBS}$, the $\mathrm{iH}$ technique is used during FLACS to initially groove and split the lens, followed by dislocating/vacuuming of the intracapsular gas (degassing). This reduces the intracapsular volume, making it possible to bypass the front and back of the intracapsular lens so as to not trap any irrigation fluid (avoiding the "irrigation trap") within the intracapsular space prior to performing the hydrodissection. As a result, this procedure can help to avoid the "lens-capsulotomy block" that could then develop into intraoperative CBS. The surgical procedure is described in the following text.

After completion of the initial steps of FLACS, which include laser irradiation for capsulotomy, lens fragmentation, and quadrants incision, and ophthalmic viscosurgical device (OVD) injection, the phaco tip is then inserted into the anterior chamber without $\mathrm{mH}$ (Figure 2A). After the anterior cortex is vacuumed by using the phaco tip within the possible range, a conventional deep vertical groove is 
sculpted in accordance with the Divide and Conquer method (Figure 2B). Subsequently, the vertical groove sculpture is sequentially cracked at both ends and at the center of the vertical groove sculpture by the tip and the hook, which bypasses the front and back of the intracapsular lens, thereby releasing the majority of the intracapsular gas with dislocation toward the anterior chamber (Figure 2C and D). The dislocated intracapsular gas can then be aspirated, which leads to a reduction in the intracapsular volume and ICP (degassing) (Figure 2E and F).

\section{Phaco-sleeve iH technique}

After bypassing the front and back of the intracapsular lens, which reduces the intracapsular volume, posterior $\mathrm{iH}$ is performed. During this step, the phaco tip and hook are kept on the base of the middle depth wall of the trench, which is referred to as cross action. During the posterior $\mathrm{iH}$, one of the sleeve side holes is directed toward the posterior capsule for the purpose of intentionally vacuuming the intraocular fluid with the phaco tip in order to induce the irrigation flow jet from the sleeve side hole into the posterior cortical-capsular layers (Figure 3A). The irrigation jet from the sleeve hole is then used to cleave the posterior cortical-capsular layer (Figure 3B and C). By repeating this method at several different points along the vertical groove sculpture, along with further repetitions after inverting the tip and hook position (referred to as non-cross action), this makes it possible to achieve a complete posterior cortical-capsular hydrodissection. In soft cataract surgery cases, however, this stage is not required. Furthermore, if the anterior cortical-capsular connection can also be separated by only using posterior $\mathrm{iH}$, it is not necessary to perform anterior $\mathrm{iH}$.

If needed, anterior $\mathrm{iH}$ is performed after the posterior $\mathrm{iH}$ (Figure 3D). After directly placing the irrigation jet from one of the sleeve side holes just inside of the capsulotomy edge so that the irrigation can pass from the sleeve hole through and under the anterior capsule, the phaco tip intentionally vacuums the intraocular fluid for the purpose of performing the anterior cortical-capsular hydrodissection. The surgeon can confirm the completion of cleavage by attempting to rotate using hook. After completion of the cortical-capsular separation, the lens can then be circumferentially rotated (Figure 3E and F). The supplementary videos present the technique in detail (see Videos S1 and $\underline{\mathrm{S} 2}$ for the surgical technique).

The remainder of the procedure, including the cataract removal and intraocular lens (IOL) implantation, is then performed in a normal manner.

\section{Femtosecond laser instrument parameters}

We used the femtosecond laser surgical system, CATALYS (Johnson \& Johnson Surgical Vision Inc., Santa Ana, CA, USA), which includes laser irradiation for capsulotomy, lens fragmentation, and quadrants (segmentation-softening spacing, 200-550 $\mu \mathrm{m}$; grid spacing, 200-350 $\mu \mathrm{m}$; segmentation repetitions, 1-5 times; horizontal spot spacing, $10 \mu \mathrm{m}$; vertical spot spacing, $40 \mu \mathrm{m}$; anterior pulse energy, $1-10 \mu \mathrm{J}$; and posterior pulse energy, $10 \mu \mathrm{J}$ ).

\section{Phacoemulsification instrument parameters}

To ensure that the irrigation jet power is sufficient for achieving an effective cortical-capsular cleavage, the phaco instrument settings for the $\mathrm{iH}$ are very important (Table 1). The four different phaco machines compared in this study were as follows: 1) the Whitestar Signature PRO (Johnson \& Johnson Surgical Vision Inc.), which used a vacuum system, Venturi pump; vacuum pressure, $160 \mathrm{mmHg}$; irrigation pressure, $60 \mathrm{~cm} \mathrm{H}_{2} \mathrm{O}$; an ultrasound power of zero; and a $20 \mathrm{G}$ diameter phaco tip with a sleeve, 2) the Signature (Johnson \& Johnson Surgical Vision Inc.), which used a vacuum system, Venturi pump; vacuum pressure, $130 \mathrm{mmHg}$; irrigation pressure, $60 \mathrm{~cm} \mathrm{H_{2 }} \mathrm{O}$; an ultrasound power of zero; and a $20 \mathrm{G}$ diameter phaco tip with a sleeve, 3) the Centurion Vision System (Alcon Laboratories, Inc., Fort Worth, TX, USA), which used a vacuum system, peristaltic pump; vacuum pressure, $350 \mathrm{mmHg}$; irrigation pressure, $36 \mathrm{mmHg}$;

Table I Recommended phaco instrument settings for $\mathrm{iH}$

\begin{tabular}{lllllll}
\hline Phaco machine & Vacuum pump & $\begin{array}{l}\text { Phaco tip } \\
\text { size (G) }\end{array}$ & $\begin{array}{l}\text { Irrigation } \\
\text { pressure }\end{array}$ & $\begin{array}{l}\text { Vacuum pressure } \\
\text { (mmHg) }\end{array}$ & $\begin{array}{l}\text { Aspiration flow } \\
\text { rate (cc/min) }\end{array}$ & $\begin{array}{l}\text { Ultrasound } \\
\text { power (\%) }\end{array}$ \\
\hline Signature PRO & Venturi & 20 & $60 \mathrm{~cm} \mathrm{H}_{2} \mathrm{O}$ & 160 & 0 \\
Signature & Venturi & 20 & $60 \mathrm{~cm} \mathrm{H}_{2} \mathrm{O}$ & 130 & 0 \\
Centurion Vision System & Peristaltic & 20 & $36 \mathrm{mmg}$ & 350 & 45 & 0 \\
Constellation Vision & Venturi & 20 & $60 \mathrm{~cm} \mathrm{H}_{2} \mathrm{O}$ & 200 & 0 \\
System & & & & &
\end{tabular}

Abbreviation: $\mathrm{iH}$, irrigation-assisted hydrodissection. 
aspiration flow rate, $45 \mathrm{cc} / \mathrm{min}$, an ultrasound power of zero, and a $20 \mathrm{G}$ diameter phaco tip with an ultra-sleeve, and 4) the Constellation Vision System (Alcon Laboratories, Inc.), which used a vacuum system, Venturi pump; vacuum

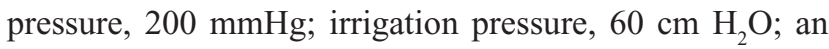
ultrasound power of zero; and a $20 \mathrm{G}$ diameter phaco tip with a micro-sleeve.

\section{Patients}

This study retrospectively reviewed the medical records of 310 consecutive eyes that underwent iH during FLACS. The surgeries were performed by two surgeons (Hiroshi Tsuneoka and Yoichiro Masuda). The study was performed in accordance with the Declaration of Helsinki ethical principles for medical research involving human subjects. The study protocol was approved by the ethics committee of Jikei University School of Medicine (registry number: 27-332 (8217)). Written informed consent was obtained from the patients to review their medical records.

\section{Experiment}

The phaco-tip sleeve and a pressure transducer were connected to the hard chamber, which simulated a closed space like the intraocular space. The phacoemulsification parameters used by the Signature PRO included a vacuum system, Venturi pump; vacuum pressure, $170 \mathrm{mmHg}$; irrigation pressure, $60 \mathrm{~cm} \mathrm{H}_{2} \mathrm{O}$; an ultrasound power of zero, and a $20 \mathrm{G}$ diameter phaco tip with a sleeve. To examine the intra-hard chamber pressure, we simulated the $\mathrm{iH}$ by vacuuming the intra-hard chamber fluid in order to induce the irrigation jet.

\section{Results}

\section{Clinical results}

The patients included 173 females and 137 males, with an average age of 70.3 years (range 19-93 years). Anterior ocular segment conditions present at surgery included pseudoexfoliation syndrome in one eye, phacodonesis in four eyes, shallow anterior chamber in 14 eyes, and post LASIK in three eyes. The ocular axial length was $<21.5 \mathrm{~mm}$ in two eyes, $21.5-26 \mathrm{~mm}$ in 234 eyes, $26-30 \mathrm{~mm}$ in 69 eyes, and $>30 \mathrm{~mm}$ in five eyes. The nucleus hardness (Emery-Little classification) was grade 1 or softer in four eyes, grade 2 in 222 eyes, grade 3 in 69 eyes, grade 4 or harder in 12 eyes, and three eyes of unknown hardness.

A polyacrylic IOL was implanted in 310 eyes, including 307 IOLs implanted in the bag and three IOLs implanted out of the bag.
Posterior capsule rupture occurred in three eyes (0.97\%). In all cases, the cause of the rupture was unrelated to the $\mathrm{iH}$, with the IOL implanted out of the bag and a final BCVA that was better than the preoperative BCVA. None of the cases exhibited any intraoperative CBS or postoperative endophthalmitis.

Preoperative BCVA was better than 20/40 in 230 eyes (74.2\%), 20/40 to $20 / 80$ in 46 eyes (14.8\%), and worse than $20 / 100$ in 34 eyes (11.0\%). Postoperative BCVA was better than $20 / 40$ in 304 eyes (98.1\%), 20/40 to $20 / 80$ in five eyes $(1.6 \%)$, and worse than $20 / 100$ in one eye $(0.3 \%)$. The low visual acuities found in six patients were related to diabetic retinopathy, macular degeneration, glaucoma, and branch retinal vein occlusion.

\section{Experimental results}

The maximum intra-hard chamber pressure was the same as the set irrigation pressure (bottle height-dependent hydrostatic pressure), whereas the intra-hard chamber pressure was reduced during the $\mathrm{iH}$ procedure.

\section{Discussion}

Roberts et $\mathrm{al}^{1}$ recommended the following intraoperative procedures for avoiding intraoperative CBS during FLACS. "1) Reduce the OVD fill to avoid overinflating the anterior chamber. 2) Decompress the anterior chamber before and during hydrodissection. 3) Decompress the lens capsule during hydrodissection. 4) Inject the hydrodissection fluid slowly and titrate. 5) Split the hemispheres prior to hydrodissection to allow the gas to come forward." When using the $\mathrm{iH}$ technique, all the five points recommended by Roberts et al can be addressed. For example, when using the iH technique during FLACS, after the initial insertion of the phaco tip following the anterior capsule removal, the phaco tip can be used to aspirate the OVD and intraocular fluid. This helps to avoid overinflating the anterior chamber and thus decompresses or stabilizes the IOP in the anterior chamber before and during the hydrodissection. Therefore, our technique is able to perform both steps 1) and 2) of the recommendations by Roberts et al. Second, since the majority of the intracapsular gas is dislocated after making the groove and splitting the lens, this means the intracapsular gas can be aspirated (degassing). As a result, the intracapsular volume and ICP will be reduced, and therefore, the front and back of the intracapsular lens can be bypassed prior to performing the hydrodissection. Thus, this step corresponds to points 3) and 5) of the recommendations by Roberts et al (Figure $5 \mathrm{~A}$ and $\mathrm{B}$ ). Third, the $\mathrm{iH}$ technique uses the phaco tip to intentionally vacuum 


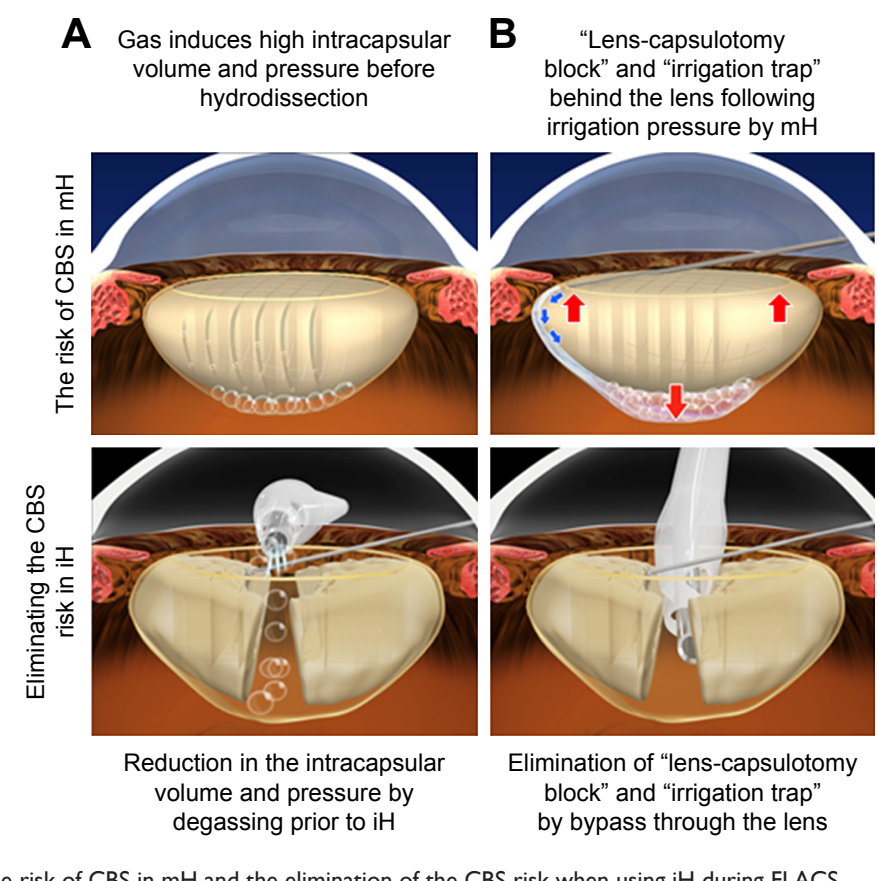

C Pressurization during $\mathrm{mH}$
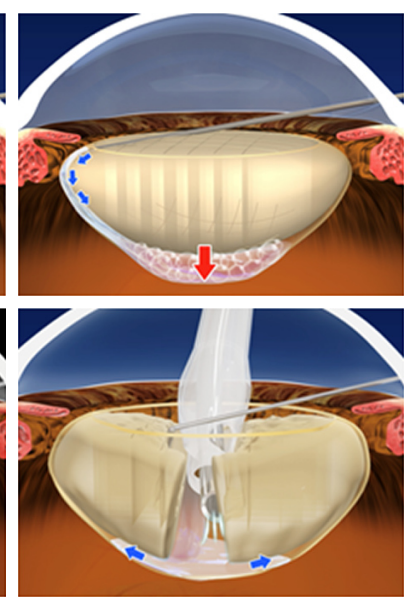

Depressurization during $\mathrm{iH}$ by controlling irrigation jet via foot pedal modulation

Figure $\mathbf{5}$ (A-C) The risk of $\mathrm{CBS}$ in $\mathrm{mH}$ and the elimination of the $\mathrm{CBS}$ risk when using iH during FLACS.

Abbreviations: CBS, capsular block syndrome; FLACS, femtosecond laser-assisted cataract surgery; iH, irrigation-assisted hydrodissection; mH, manual hydrodissection.

the intraocular fluid in order to induce the irrigation jet from the phaco tip sleeve side hole for cortical-capsular cleavage. Since the jet power is dependent on the aspiration flow rate that can be modulated by a foot pedal, we can inject the hydrodissection fluid and titrate the volume via the use of the foot pedal modulation, thereby making it possible to reduce the ICP during hydrodissection. Moreover, we confirmed that the pressure was reduced during the $\mathrm{iH}$ procedure in experiment using bottle height-dependent irrigation pressure phaco machine. This step corresponds to points 3) and 4) of the recommendations by Roberts et al (Figure 5C).

\section{Conclusion}

This study demonstrated the utility of using $\mathrm{iH}$ during FLACS in 310 consecutive cases, with all the procedures performed without intraoperative CBS. The technique has an advantage not only of intracapsular depressurization during hydrodissection but also that the use of special instrument is unnecessary. Thus, the use of this $\mathrm{iH}$ technique appears to be beneficial for patients, as it can prevent CBS during FLACS.

\section{Acknowledgment}

The authors thank Noriko Kato and Akiko Kiriyama for their helpful suggestions regarding the use of the phaco-sleeve irrigation-assisted hydrodissection technique.

\section{Disclosure}

The authors report no conflicts of interest in this work.

\section{References}

1. Roberts TV, Sutton G, Lawless MA, Jindal-Bali S, Hodge C. Capsular block syndrome associated with femtosecond laser-assisted cataract surgery. J Cataract Refract Surg. 2011;37(11):2068-2070.

2. Yoe R. Hydrorupture of posterior capsule in femtosecond-laser cataract surgery [letter]. J Cataract Refract Surg. 2012;38:730.

3. Hurvitz LM. Posterior capsular rupture at hydrodissection. J Cataract Refract Surg. 1991;17(6):866.

4. Ota I, Miyake S, Miyake K. Dislocation of the lens nucleus into the vitreous cavity after standard hydrodissection. Am J Ophthalmol. 1996;121(6):706-708.

5. Miyake K, Ota I, Ichihashi S, Miyake S, Tanaka Y, Terasaki H. New classification of capsular block syndrome. J Cataract Refract Surg. 1998;24(9):1230-1234.

6. Yeoh R. The 'pupil snap' sign of posterior capsule rupture with hydrodissection in phacoemulsification. Br J Ophthalmol. 1996;80(5):486.

7. Yeoh R, Theng J. Capsular block syndrome and pseudoexpulsive hemorrhage. J Cataract Refract Surg. 2000;26(7):1082-1084.

8. Roberts TV, Lawless M, Bali SJ, Hodge C, Sutton G. Surgical outcomes and safety of femtosecond laser cataract surgery: a prospective study of 1500 consecutive cases. Ophthalmology. 2013;120(2):227-233.

9. Masuda Y, Iwaki H, Kato N, Takahashi G, Oki K, Tsuneoka H. Irrigation dynamic pressure-assisted hydrodissection during cataract surgery. Clin Ophthalmol. 2017;11:323-328.

10. Masuda Y, Oki K, Iwaki H, Okamoto T, Tsuneoka H. Use of the phaco tip technique for lens cleavage and removal during cataract surgery. Clin Ophthalmol. 2016;10:1925-1929.

11. Masuda Y, Tsuneoka H. Hydrodissection-free phacoemulsification surgery: mechanical cortical cleaving dissection. $J$ Cataract Refract Surg. 2014;40(8):1327-1331. 
Clinical Ophthalmology

\section{Publish your work in this journal}

Clinical Ophthalmology is an international, peer-reviewed journal covering all subspecialties within ophthalmology. Key topics include: Optometry; Visual science; Pharmacology and drug therapy in eye diseases; Basic Sciences; Primary and Secondary eye care; Patien Safety and Quality of Care Improvements. This journal is indexed on

PubMed Central and CAS, and is the official journal of The Society of Clinical Ophthalmology (SCO). The manuscript management system is completely online and includes a very quick and fair peer-review system, which is all easy to use. Visit http://www.dovepress.com/ testimonials.php to read real quotes from published authors. 